\title{
Impact of polymorphisms in WFS1 on prediabetic phenotypes in a population-based sample of middle-aged people with normal and abnormal glucose regulation
}

\author{
T. Sparsø • G. Andersen • A. Albrechtsen • \\ T. Jørgensen • K. Borch-Johnsen • A. Sandbak • \\ T. Lauritzen • J. Wasson • M. A. Permutt • B. Glaser • \\ S. Madsbad • O. Pedersen • T. Hansen
}

Received: 8 March 2008/Accepted: 5 May 2008/Published online: 21 June 2008

(C) Springer-Verlag 2008

\begin{abstract}
Aim/hypothesis Recently, variants in WFS1 have been shown to be associated with type 2 diabetes. We aimed to examine metabolic risk phenotypes of WFS1 variants in glucosetolerant people and in individuals with abnormal glucose regulation.

Methods The type 2 diabetes-associated WFS1 variant rs734312 (His611Arg) was studied in the population-based Inter99 cohort involving 4,568 glucose-tolerant individuals and 1,471 individuals with treatment-naive abnormal glucose regulation, and in an additional 3,733 treated type 2 diabetes patients.
\end{abstract}

Electronic supplementary material The online version of this article (doi:10.1007/s00125-008-1064-2) contains supplementary material, which is available to authorised users.

T. Sparsø $(\bowtie) \cdot$ G. Andersen $\cdot$ K. Borch-Johnsen · O. Pedersen •

T. Hansen

Steno Diabetes Center,

Niels Steensens Vej 1, NLC2.13, DK-2820 Gentofte,

Copenhagen, Denmark

e-mail: tspr@steno.dk

\section{A. Albrechtsen}

Department of Biostatistics,

University of Copenhagen,

Copenhagen, Denmark

\section{T. Jørgensen $\cdot \mathrm{K}$. Borch-Johnsen}

Research Centre for Prevention and Health,

Glostrup University Hospital,

Glostrup, Denmark

K. Borch-Johnsen • O. Pedersen

Faculty of Health Science, University of Aarhus,

Aarhus, Denmark
Results The WFS1 rs734312 showed a borderline significant association with type 2 diabetes with directions and relative risks consistent with previous reports. In individuals with abnormal glucose regulation, the diabetogenic risk A allele of rs734312 was associated in an allele-dependent manner with a decrease in insulinogenic index $(p=0.025)$ and decreased 30-min serum insulin levels $(p=0.047)$ after an oral glucose load. In glucose-tolerant individuals the same allele was associated with increased fasting serum insulin concentration $(p=0.019)$ and homeostasis model assessment of insulin resistance (HOMA-IR; $p=0.026$ ). To study the complex interaction of WFS1 rs734312 on insulin release

\author{
A. Sandbæk $\cdot$ T. Lauritzen \\ Department of General Practice, University of Aarhus, \\ Aarhus, Denmark \\ J. Wasson · M. A. Permutt \\ Metabolism and Lipid Research Division, \\ Washington University School of Medicine, \\ St Louis, MO, USA \\ B. Glaser \\ Hadassah-Hebrew University Medical School, \\ Jerusalem, Israel \\ S. Madsbad \\ Hvidovre University Hospital, \\ Hvidovre, Denmark \\ O. Pedersen \\ Faculty of Health Science, University of Copenhagen, \\ Copenhagen, Denmark
}


and insulin resistance we introduced Hotelling's $T^{2}$ test. Assuming bivariate normal distribution, we constructed standard error ellipses of the insulinogenic index and HOMA-IR when stratified according to glucose tolerance status around the means of each WFS1 rs734312 genotype level. The interaction term between individuals with normal glucose tolerance and abnormal glucose regulation on the insulinogenic index and HOMA-IR was significantly associated with the traits $(p=0.0017)$.

Conclusions/interpretation Type 2 diabetes-associated risk alleles of WFS1 are associated with estimates of a decreased pancreatic beta cell function among middle-aged individuals with abnormal glucose regulation.

Keywords ADDITION - Beta cell · Case-control study . Genetic association · Insulin resistance · Inter99 .

Single-nucleotide polymorphism · Type 2 diabetes

\begin{tabular}{|c|c|}
\hline \multicolumn{2}{|c|}{ Abbreviations } \\
\hline ER & endoplasmic reticulum \\
\hline HOMA-IR & $\begin{array}{l}\text { homeostasis model assessment of insulin } \\
\text { resistance }\end{array}$ \\
\hline IFG & impaired fasting glycaemia \\
\hline IGT & impaired glucose tolerance \\
\hline LD & linkage disequilibrium \\
\hline SNP & single-nucleotide polymorphism \\
\hline WFS1 & Wolfram syndrome 1 (wolframin) \\
\hline
\end{tabular}

\section{Introduction}

A study by Sandhu et al. reported the results of genotyping 1,536 single-nucleotide polymorphisms (SNPs) in 84 candidate genes regulating pancreatic beta cell development, growth, function or survival [1]. In a pooled analysis involving six UK studies and one study of an Ashkenazi Jewish population including a total of 9,533 type 2 diabetic patients and 11,389 controls the authors showed that four common variants (rs10010131, rs6446482, rs752854 and rs734312) of the gene encoding wolframin 1 (WFS1) were associated with type 2 diabetes. Two independent studies have investigated these initial findings [1]. The first study by Franks et al. [2] replicated the nominal association of the four WFS1 SNPs with type 2 diabetes, whereas the overall conclusion of the second study by Florez et al. [3] was that no association with type 2 diabetes could be observed. However, a thorough phenotype association analysis was performed to elucidate a possible prediabetic phenotype. The study consisted of 3,530 US individuals at high risk of developing type 2 diabetes (on the basis of overweight, increased fasting plasma glucose and impaired glucose tolerance [IGT]) and investigated the impact of WFS1 SNPs (including rs10010131 and rs734312) in relation to insulin sensitivity and beta cell function. Interestingly, carriers of the type 2 diabetes risk A allele of rs 734312 were characterised by an allele-dependent reduction in insulin release and a concomitant increase in insulin sensitivity [3]. Indeed, the notion that WFS1 is a true type 2 diabetes gene seems to be established, although some further validation of an intermediate prediabetic phenotype causing the observed diabetes association is still needed.

WFS1 encodes an 890 amino acid membrane glycoprotein located in the endoplasmic reticulum (ER). It is ubiquitously expressed in many organs including the brain and pancreas, with weak signals detected in liver, skeletal muscle and kidney [4, 5]. Mutations in WFS1 are known to cause the Wolfram syndrome, which is an autosomal recessive disorder clinically defined by diabetes insipidus, non-autoimmune diabetes mellitus with juvenile onset, optic atrophy and deafness $[5,6]$. Disruption of $W f S 1$ in mice resulted in progressive glucose intolerance and concomitant insulin deficiency. In this animal model, beta cell death occurred by an accelerated process of apoptosis; similarly, increased levels of markers reflecting ER stress were also demonstrated [7-9]. In line with the outcome of studies in mice, previous and statistically undersized human studies have indicated that variation in WFS1 may be associated with type 1 diabetes mellitus [10], type 2 diabetes mellitus [11] and a combination of diabetes mellitus and deafness [12, 13]. Moreover, two patients with the Wolfram syndrome were reported to be without insulin-producing beta cells [14].

The aim of the present study was to establish possible metabolic risk phenotypes of the type 2 diabetes-associated A-allele of rs734312 (His611Arg), the G allele of rs6446482 and the $\mathrm{G}$ allele of rs10010131 in a large population-based cohort of middle-aged glucose-tolerant individuals as well as in treatment-naive individuals with abnormal glucose regulation. Furthermore, we aimed to confirm the recently reported [1-3] diabetogenic impact of the WFS1 risk alleles in our Danish case-control study involving 3,844 type 2 diabetes patients and 4,225 glucose-tolerant control participants.

\section{Methods}

Study population Three WFS1 polymorphisms (rs10010131, rs6446482 and rs734312) were genotyped in 9,772 Danes involving: (1) the population-based Inter99 cohort (ClinicalTrial.gov ID no NCT00289237) of middle-aged individuals sampled at Research Centre for Prevention and Health $(n=6,039)$ [15]; (2) type 2 diabetic patients sampled through the outpatient clinic at Steno Diabetes Center $(n=2,107)$; and (3) the screen-detected type 2 diabetes patients from the Danish ADDITION screening cohort (ClinicalTrials.gov NCT00237549) sampled through Department of General Practice at University of Aarhus $(n=1,626)$ [16]. Study 
group 1 underwent a standard $75 \mathrm{~g}$ OGTT. Informed written consent was obtained from all participants before participation. The study was approved by the Ethical Committee of Copenhagen County and was in accordance with the principles of the Helsinki Declaration. Normal glucose tolerance, impaired fasting glycaemia (IFG), IGT and type 2 diabetes were defined according to WHO [17].

Biochemical and anthropometrical measurements Height and body weight were measured in light indoor clothes and without shoes; BMI was calculated as weight $(\mathrm{kg}) /($ height $[\mathrm{m}])^{2}$. Blood samples were drawn after a $12 \mathrm{~h}$ overnight fast. Plasma glucose was analysed by a glucose oxidase method (Granutest, Merck, Darmstadt, Germany). $\mathrm{HbA}_{1 \mathrm{c}}$ was measured by ion-exchange high-performance liquid chromatography (normal reference range: 4.1-6.4\%) and serum insulin, excluding des(31, 32) and intact proinsulin, was measured using a kit (AutoDELFIA insulin kit; Perkin-Elmer, Wallac, Turku, Finland). Insulinogenic index insulin $_{\text {was }}$ calculated as (serum insulin at $30 \mathrm{~min}[\mathrm{pmol} / \mathrm{l}]$-fasting serum insulin [pmol/1])/plasma glucose at $30 \mathrm{~min}(\mathrm{mmol} / \mathrm{l})$, which is a surrogate estimate of the initial oral glucose-elicited insulin release. Homeostasis model assessment of insulin resistance (HOMA-IR) was calculated as described previously [18].

Genotyping The WFS1 polymorphisms (rs10010131, rs6446482 and rs734312) were genotyped using Taqman allelic discrimination (KBioscience, Hoddesdon, UK).
Discordance between 941 random duplicate samples was $0.1 \%$ and the genotyping success rate was $>97 \%$ for all SNPs. All genotype groups obeyed Hardy-Weinberg equilibrium.

Statistical analysis Fisher's exact test and logistic regression adjusted for sex, age and BMI were applied to examine differences in allele frequencies and genotype distributions between affected and unaffected participants. General linear statistics was used to test quantitative variables for differences between genotype groups of glucosetolerant individuals and treatment-naive individuals with abnormal glucose regulation (IFG, IGT and screendetected type 2 diabetes). The multivariate method, Hotelling $T^{2}$ [19], was applied to test the simultaneous effect of rs 734312 on two traits of insulin release and insulin sensitivity.

All analyses were performed using RGui version 2.6.0 (http://www.r-project.org). We considered $p$ values below 0.05 after 5,000 permutations using the statistical software BLOSSOM (available from the US Geological Survey; http:// www.fort.usgs.gov/products/software/blossom/blossom.asp) to be significant. Since type 2 diabetic patients included in the case-control study were ascertained from three different study populations, we evaluated their possible heterogeneity by comparing allele frequencies of the three WFSlvariants in type 2 diabetes patients from the different subgroups. No evidence of heterogeneity was observed using Pearson's $\chi^{2}$ test for count data.

Table 1 Association studies of rs10010131 and rs734312 of WFS1 in 3,844 type 2 diabetic patients and 4,225 glucose-tolerant control participants

\begin{tabular}{|c|c|c|c|c|c|c|}
\hline & \multicolumn{3}{|c|}{ rs10010131 (genotype risk allele G) } & \multicolumn{3}{|c|}{ rs734312 (genotype risk allele A) } \\
\hline & GG & GA & AA & AA & GA & GG \\
\hline \multicolumn{7}{|c|}{ Genotype distribution, $n(\%)$} \\
\hline Glucose tolerance & $1,373(33)$ & $2,105(50)$ & 747 (18) & $1,094(27)$ & $2,054(50)$ & $960(23)$ \\
\hline Type 2 diabetes & $1,326(34)$ & $1,878(49)$ & $640(17)$ & $1,098(28)$ & $1,920(50)$ & $823(22)$ \\
\hline \multicolumn{7}{|c|}{ Allele frequency model ${ }^{\mathrm{a}}$} \\
\hline OR $(95 \% \mathrm{CI})$ & $0.94(0.88-1.00)$ & & & $0.93(0.87-0.99)$ & & \\
\hline$p$ value & 0.053 & & & 0.024 & & \\
\hline \multicolumn{7}{|c|}{ Genotype frequency model ${ }^{\mathrm{a}}$} \\
\hline$p$ value & 0.13 & & & 0.072 & & \\
\hline \multicolumn{7}{|l|}{ Additive $^{\mathrm{b}}$} \\
\hline OR $(95 \% \mathrm{CI})$ & $0.95(0.86-1.05)$ & & & $0.91(0.83-1.00)$ & & \\
\hline$p$ value & 0.32 & & & 0.056 & & \\
\hline \multicolumn{7}{|l|}{ Dominant ${ }^{\mathrm{b}}$} \\
\hline OR $(95 \% \mathrm{CI})$ & $0.90(0.78-1.03)$ & & & $0.85(0.73-0.99)$ & & \\
\hline$p$ value & 0.13 & & & 0.040 & & \\
\hline \multicolumn{7}{|l|}{ Recessive $^{\mathrm{b}}$} \\
\hline OR $(95 \% \mathrm{CI})$ & $1.01(0.84-1.20)$ & & & $0.92(0.78-1.08)$ & & \\
\hline$p$ value & 0.95 & & & 0.30 & & \\
\hline
\end{tabular}

Data are number of participants with each genotype (\% of each group), risk allele frequencies in per cent (95\% CI) and OR (95\% CI)

${ }^{a}$ Differences in allele frequencies and genotype distribution not adjusted for age, sex or BMI were calculated using Fisher's exact test

${ }^{\mathrm{b}}$ Logistic regression applying an additive, dominant or recessive model adjusted for age, sex and BMI 
To investigate whether the effect of the alleles differed between individuals with different glucose tolerance status, we included an interaction term between glucose tolerance status and the genotype of interest in the linear model besides the main effects:

$$
\begin{aligned}
y_{\text {trait }}= & \mu+\alpha X_{\mathrm{add}}+\beta X_{\mathrm{gts}}+\gamma X_{\mathrm{add}} X_{\mathrm{gts}}+\delta X_{\mathrm{sex}}+ \\
& \in X_{\mathrm{age}}+\zeta X_{\mathrm{BMI}}
\end{aligned}
$$

In this model, we assumed an additive effect for the genotypes (i.e. $X_{\text {add }}$ coded as the number of minor alleles) and treated the glucose tolerance status $\left(X_{\mathrm{gts}}\right)$ as a binary vector indicating whether individuals belonged to the group of glucose-tolerant individuals (coded as 0 ) or abnormal glucose regulation (coded as 1). The same procedure was also performed for a bivariate distribution of two traits

Table 2 Anthropometric and metabolic characteristics of 5,384 treatment-naive Danish individuals from the Inter99 cohort stratified according to

\begin{tabular}{|c|c|c|c|c|c|}
\hline Characteristic & $\mathrm{A} / \mathrm{A}$ & $\mathrm{A} / \mathrm{G}$ & $\mathrm{G} / \mathrm{G}$ & $p_{\text {add }}$ & $p_{\text {int }}$ \\
\hline \multicolumn{6}{|l|}{ Normal glucose tolerance } \\
\hline$n$ (men/women) & $1,091(495 / 596)$ & $2,050(962 / 1088)$ & $958(445 / 513)$ & & \\
\hline Age (years) & $45 \pm 8$ & $45 \pm 8$ & $45 \pm 8$ & & \\
\hline BMI $\left(\mathrm{kg} / \mathrm{m}^{2}\right)$ & $25.5 \pm 4.0$ & $25.5 \pm 4.1$ & $25.6 \pm 4.1$ & 0.56 & \\
\hline \multicolumn{6}{|l|}{ Serum insulin $(\mathrm{pmol} / \mathrm{l})$} \\
\hline Fasting & $39 \pm 24$ & $38 \pm 23$ & $37 \pm 23$ & 0.019 & \\
\hline $30 \mathrm{~min}$ post-OGTT & $290 \pm 190$ & $291 \pm 178$ & $278 \pm 156$ & 0.085 & \\
\hline 120 min post-OGTT & $174 \pm 145$ & $169 \pm 126$ & $166 \pm 130$ & 0.12 & \\
\hline \multicolumn{6}{|l|}{ Plasma glucose $(\mathrm{mmol} / \mathrm{l})$} \\
\hline Fasting & $5.3 \pm 0.4$ & $5.3 \pm 0.4$ & $5.3 \pm 0.4$ & 0.77 & \\
\hline 30-min post-OGTT & $8.1 \pm 1.6$ & $8.2 \pm 1.5$ & $8.2 \pm 1.5$ & 0.78 & \\
\hline 120-min post-OGTT & $5.5 \pm 1.1$ & $5.5 \pm 1.1$ & $5.5 \pm 1.1$ & 0.53 & \\
\hline \multicolumn{6}{|l|}{ Serum C-peptide (pmol/l) } \\
\hline Fasting & $545 \pm 208$ & $537 \pm 209$ & $534 \pm 220$ & 0.030 & \\
\hline 30-min post-OGTT & $1,984 \pm 668$ & $1,988 \pm 710$ & $1,936 \pm 666$ & 0.039 & \\
\hline 120-min post-OGTT & $2,072 \pm 804$ & $2,064 \pm 794$ & $2,025 \pm 796$ & 0.12 & \\
\hline HOMA-IR & $9.2 \pm 5.8$ & $8.9 \pm 5.7$ & $8.7 \pm 5.5$ & 0.026 & \\
\hline Insulinogenic index ${ }_{\text {Insulin }}(\mathrm{pmol} / \mathrm{mmol})$ & $31.2 \pm 20.9$ & $31.4 \pm 19.9$ & $30.0 \pm 18.1$ & 0.075 & \\
\hline \multicolumn{6}{|l|}{ Abnormal glucose tolerance ${ }^{\mathrm{a}}$} \\
\hline$n$ (men/women) & $359(224 / 135)$ & $642(385 / 257)$ & $284(166 / 118)$ & & \\
\hline Age (years) & $50 \pm 8$ & $49 \pm 7$ & $49 \pm 8$ & & \\
\hline BMI $\left(\mathrm{kg} / \mathrm{m}^{2}\right)$ & $28.3 \pm 4.9$ & $28.3 \pm 5.3$ & $28.6 \pm 5.2$ & 0.49 & 0.83 \\
\hline \multicolumn{6}{|l|}{ Serum insulin $(\mathrm{pmol} / \mathrm{l})$} \\
\hline Fasting & $54 \pm 33$ & $58 \pm 37$ & $55 \pm 33$ & 1.0 & 0.28 \\
\hline 30 min post-OGTT & $291 \pm 181$ & $311 \pm 224$ & $333 \pm 214$ & 0.047 & 0.0088 \\
\hline 120 min post-OGTT & $370 \pm 304$ & $364 \pm 320$ & $390 \pm 354$ & 1.0 & 0.18 \\
\hline \multicolumn{6}{|l|}{ Plasma glucose $(\mathrm{mmol} / \mathrm{l})$} \\
\hline Fasting & $6.3 \pm 1.35$ & $6.2 \pm 1.0$ & $6.2 \pm 1.2$ & 0.38 & 0.27 \\
\hline $30 \mathrm{~min}$ post-OGTT & $10.2 \pm 1.9$ & $10.3 \pm 1.9$ & $10.2 \pm 1.7$ & 0.60 & 0.35 \\
\hline $120 \mathrm{~min}$ post-OGTT & $8.5 \pm 2.9$ & $8.4 \pm 2.9$ & $8.2 \pm 2.3$ & 0.36 & 0.70 \\
\hline \multicolumn{6}{|l|}{ Serum C-peptide (pmol/l) } \\
\hline Fasting & $756 \pm 331$ & $772 \pm 348$ & $768 \pm 340$ & 0.97 & 0.56 \\
\hline 30 min post-OGTT & $2,039 \pm 762$ & $2,059 \pm 823$ & $2,147 \pm 751$ & 0.17 & 0.021 \\
\hline $120 \mathrm{~min}$ post-OGTT & $3,130 \pm 1201$ & $3,054 \pm 1223$ & $3,159 \pm 1238$ & 0.84 & 0.19 \\
\hline HOMA-IR & $15.3 \pm 10.1$ & $16.2 \pm 11.6$ & $15.3 \pm 9.9$ & 0.81 & 0.34 \\
\hline Insulinogenic index ${ }_{\text {Insulin }}(\mathrm{pmol} / \mathrm{mmol})$ & $23.5 \pm 16.0$ & $25.1 \pm 19.5$ & $27.5 \pm 20.5$ & 0.025 & 0.0025 \\
\hline
\end{tabular}
WFS1 rs734312 genotype and glucose tolerance status

Data are means \pm SD. Values of serum insulin and values derived from serum insulin variables were logarithmically transformed before statistical analysis. All analyses were made using an additive model.

Sex and glucose tolerance status were considered as discrete factors and age, genotype and BMI as continuous covariates.

Calculated $p$ values were adjusted for age, sex and BMI (where appropriate): the $p$ values for an interaction ( $1 d f$ ) between glucose tolerance status and genotype, as described in the Methods, are denoted $p_{\text {int }}$.

The insulinogenic index was calculated as fasting serum insulin (pmol/l) subtracted from 30 min post-OGTT serum insulin (pmol/l) and divided by $30 \mathrm{~min}$ post-OGTT plasma glucose (mmol/1).

HOMA-IR was calculated as previously described [18]

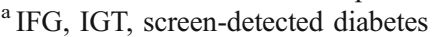


where the significance of the interaction was assessed by the multivariate method Hotelling $T^{2}$ test.

\section{Results}

We estimated the linkage disequilibrium (LD) pattern assessed by $r^{2}$ of the three WFS1 SNPs (rs10010131, rs6446482 and rs734312) (Electronic supplementary material [ESM] Fig. 1). As the LD between rs10010131 and rs6446482 was high $\left(r^{2}>0.95\right)$, only rs734312 and rs10010131 were analysed in the present paper. The minor allele frequency in the population-based Inter99 cohort was $42 \%$ for rs 10010131 and $48 \%$ for rs 734312 .

In the present Danish case-control study, the WFS1 rs734312 showed a borderline significant association with type 2 diabetes with a direction and a relative risk consistent with previous reports (Table 1).

In order to investigate whether intermediate prediabetic phenotypes are associated with the reported WFS1 type 2 diabetes risk alleles, we investigated metabolic traits related to type 2 diabetes in 4,568 glucose-tolerant individuals and in 1,471 treatment-naive individuals with abnormal glucose regulation (IFG, IGT and screen-detected type 2 diabetes). In individuals with abnormal glucose regulation, the diabetogenic risk A allele of rs 734312 was associated in an allele-dependent manner with a decreased insulinogenic index $(p=0.025)$ and a decreased 30-min serum insulin level $(p=0.047)$ after an oral glucose load, whereas in glucosetolerant individuals the same allele was associated with increased fasting serum insulin $(p=0.019)$, fasting serum C-peptide $(p=0.030)$ and HOMA-IR $(p=0.026$; Table 2$)$. Similar results were found for the diabetes risk allele of rs10010131 (ESM Table 1).

We searched results of available web-based genomewide association studies of WFS1 SNPs and their potential relationships to traits associated with type 2 diabetes such as fasting serum insulin, 120 min post-OGTT serum insulin and insulinogenic index, as well as estimates of insulin resistance. However, no significant associations with any of the ten SNPs genotyped in WFSI were observed [20].

We also examined for interaction between glucose tolerance status and genotype by applying a linear model for quantitative indices of pancreatic beta cell function. In this analysis, we demonstrated a significant interaction of rs 734312 with traits related to beta cell function (30-min serum insulin $p=0.0088$, insulinogenic index $p=0.0025$ ), which implies a larger effect size of WFS1 variants for individuals with abnormal glucose regulation than for glucose tolerant individuals (Table 2, ESM Table 1). In glucose-tolerant people, the effect size on beta cell-related traits such as the insulinogenic index seems to be vague, i.e. $2.2 \%(95 \%$ CI $[-4.7,0.2]$ reduction per allele, $p=0.075)$, whereas in the group of individuals with abnormal glucose regulation the impact increases considerably, i.e. $6.5 \%$ $(95 \%$ CI $[0.8,12.1]$ increase per allele, $p=0.025)$. Similar results were observed for $30 \mathrm{~min}$ serum insulin and Cpeptide after an oral glucose load (ESM Table 2).

The interplay between WFS1 rs 734312 genotype, insulin release and insulin resistance in relation to glucose tolerance status in the Inter99 cohort is shown in Fig. 1. It appears that there is a glucose tolerance interaction affecting the two correlated traits $(p=0.0017)$ and that the largest effect is on insulin release as assessed by the insulinogenic index in A allele carriers with abnormal glucose regulation. With regard to rs10010131, an interaction of glucose tolerance status on the insulinogenic index and HOMA-IR was also found to be statistically significant ( $p=0.0027$, data not shown).

\section{Discussion}

In the present study, we assessed the impact of the type 2 diabetes risk alleles of rs734312 and rs10010131 on prediabetic phenotypes that may explain the previously reported diabetogenic impact of these variants [1-3]. In subgroups of a population-based cohort of middle-aged people with normal glucose tolerance and treatment-naive abnormal

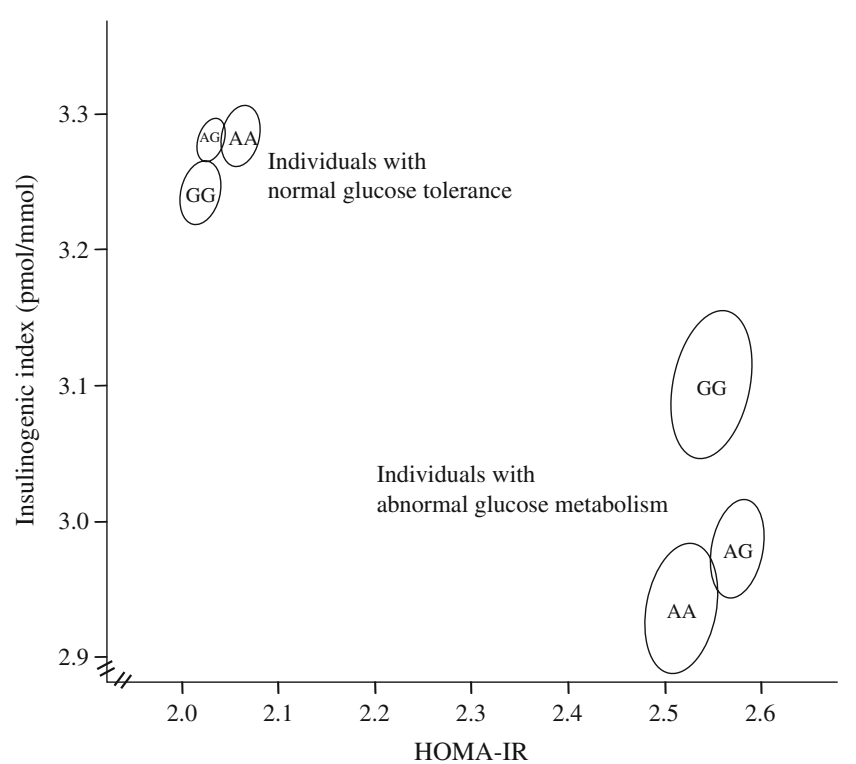

Fig. 1 Standard error of the mean of the insulinogenic index and HOMA-IR stratified according to glucose tolerance status and rs734312 genotype in middle-aged individuals from the Danish Inter99 cohort. Assuming bivariate normal distribution, we constructed standard error ellipses around the means of each genotype level. The interaction term between individuals with normal glucose tolerance (AA: 980, AG: 1898, GG: 874) and abnormal glucose regulation (AA: 355, AG: 632, GG: 286) on the insulinogenic index and HOMA-IR was significantly associated with the traits $(p=0.0017)$ when applying Hotelling $T^{2}$ 
glucose regulation we found that the effect size of WFS1 diabetes risk alleles differed depending on glucose-tolerance status. In the individuals with abnormal glucose regulation, defined as IFG, IGT or screen-detected and untreated type 2 diabetes, the reported WFS1 diabetes risk alleles were significantly associated with impaired insulin release as assessed by insulinogenic index ( $6.5 \%$ per allele), whereas the same alleles in glucose-tolerant individuals were associated with increased fasting serum insulin and HOMA-IR. To date, two studies [2, 3] have investigated the association between WFS1 variants and type 2 diabetes suggested by Sandhu et al. [1]; however, only one study has considered the underlying prediabetic phenotype [3]. The latter study involved 3,530 US individuals of various ethnicities, all at high risk of developing type 2 diabetes. The authors of that report demonstrated an increased post-oral glucose load insulin release as estimated by the insulinogenic index and a decreased insulin sensitivity index for the reported protective G-allele of rs734312. Thus, these results [3] on insulin release are in line with our findings in Danish middle-aged participants with untreated abnormal glucose regulation. However, due to the modest effect size of the WFS1 variants, both reported results should be considered as preliminary findings and obviously they will need to be replicated in independent and statistically powered study samples.

Taken together, the known pathophysiology of the Wolfram syndrome and the numerous studies of the comparable gene in mice suggest that the Wolfram syndrome 1 (wolframin) protein (WFS1) primarily mediates its action at the pancreatic beta cell level. Specifically, it has been localised to the $E R$, indicating a role in membrane trafficking, secretion and regulation of ER calcium homeostasis, i.e. biological functions that cause ER stress if disturbed. These mechanisms have also been examined in $W f_{s} 1$-deficient mice that exhibited increased phosphorylation of RNA-dependent protein kinase-like ER kinase, chaperone gene expression and active X-box binding protein 1 levels, all of which are factors pointing to an enhanced ER stress response.

In line with the known function of WFS1, the diabetes risk allele-dependent decrease in post-oral glucose load insulin release observed in individuals with abnormal glucose regulation and not in glucose-tolerant individuals may tentatively be explained by the fact that other diabetogenic factors are operating in the group of people with abnormal glucose regulation. Thus, individuals with abnormal glucose regulation may have several stressors of their pancreatic beta cell function, including insulin resistance, proinflammation, glucotoxicity and lipotoxicity, all of which may interact with susceptibility variants in WFS1 and hereby predispose to ER stress-mediated apoptosis.
Acknowledgements This study was supported by the Danish Medical Research Council, the Danish Diabetes Association, the Gerda and Aage Haensch Foundation, the A.P. Møller Foundation for the Advancement of Medical Science, University of Copenhagen, Novo Nordisk and the European Union (InterAct, grant no. LSHMCT-2006-037197). This study also received support from The FOOD Study Group/the Danish Ministry of Food. The authors wish to thank A. Forman, I.-L. Wantzin, T. Lorentzen and M. Stendal for technical assistance, G. Lademann for secretarial support and A. Vølund for statistical advice and guidance.

Duality of interest K. Borch-Johnsen and O. Pedersen hold stock in Novo Nordisk and have received lecture fees from pharmaceutical companies. All other authors declare that there is no duality of interest associated with this manuscript.

\section{References}

1. Sandhu MS, Weedon MN, Fawcett KA et al (2007) Common variants in WFS1 confer risk of type 2 diabetes. Nat Genet 39:951-953

2. Franks PW, Rolandsson O, Debenham SL et al (2008) Replication of the association between variants in WFS1 and risk of type 2 diabetes in European populations. Diabetologia. 51:458-463

3. Florez JC, Jablonski KA, McAteer J et al (2008) Testing of diabetes-associated WFS1 polymorphisms in the Diabetes Prevention Program. Diabetologia 51:451-457

4. Hofmann S, Philbrook C, Gerbitz KD et al (2003) Wolfram syndrome: structural and functional analyses of mutant and wildtype wolframin, the WFS1 gene product. Hum Molec Genet 12: 2003-2012

5. Inoue H, Tanizawa Y, Wasson J et al (1998) A gene encoding a transmembrane protein is mutated in patients with diabetes mellitus and optic atrophy (Wolfram syndrome). Nat Genet 20:143-148

6. Strom TM, Hörtnagel K, Hofmann S et al (1998) Diabetes insipidus, diabetes mellitus, optic atrophy and deafness (DIDMOAD) caused by mutations in a novel gene (wolframin) coding for a predicted transmembrane protein. Hum Mol Genet 7:2021-2028

7. Ishihara H, Takeda S, Tamura A et al (2004) Disruption of the WFS1 gene in mice causes progressive $\beta$-cell loss and impaired stimulus - secretion coupling in insulin secretion. Hum Mol Genet 13:1159-1170

8. Riggs AC, Bernal-Mizrachi E, Ohsugi M et al (2005) Mice conditionally lacking the Wolfram gene in pancreatic islet beta cells exhibit diabetes as a result of enhanced endoplasmic reticulum stress and apoptosis. Diabetologia 48:2313-2321

9. Yamada T, Ishihara H, Tamura A et al (2006) WFS1-deficiency increases endoplasmic reticulum stress, impairs cell cycle progression and triggers the apoptotic pathway specifically in pancreatic beta-cells. Hum Mol Genet 15:1600-1609

10. Awata T, Inoue K, Kurihara S et al (2000) Missense variations of the gene responsible for Wolfram syndrome (WFS1/wolframin) in Japanese: possible contribution of the Arg456His mutation to type 1 diabetes as a nonautoimmune genetic basis. Biochem Biophys Res Commun 268:612-616

11. Minton JAL, Hattersley AT, Owen K et al (2002) Association studies of genetic variation in the WFS1 gene and type 2 diabetes in U.K. populations. Diabetes 51:1287-1290 
12. Domènech E, Gómez-Zaera M, Nunes V (2002) WFS1 mutations in Spanish patients with diabetes mellitus and deafness. Eur J Hum Genet 10:421-426

13. Eiberg H, Hansen L, Kjer B et al (2006) Autosomal dominant optic atrophy associated with hearing impairment and impaired glucose regulation caused by a missense mutation in the WFS1 gene. J Med Genet 43:435-440

14. Karasik A, O'Hara C, Srikanta S et al (1989) Genetically programmed selective islet $\beta$-cell loss in diabetic subjects with Wolfram's syndrome. Diabetes Care 12:135-138

15. Jørgensen T, Borch-Johnsen K, Thomsen TF et al (2003) A randomized non-pharmacological intervention study for prevention of ischaemic heart disease: baseline results Inter99 (1). Eur J Cardiovasc Prevention Rehab 10:377-386

16. Lauritzen T, Griffin S, Borch-Johnsen K et al (2000) The ADDITION study: proposed trial of the cost-effectiveness of an intensive multifactorial intervention on morbidity and mortality among people with type 2 diabetes detected by screening. Int $\mathrm{J}$ Obes Suppl 3:S6-S11

17. Alberti KGMM, Zimmett PZ, for a WHO consultation (1999) Definition, diagnosis and classification of diabetes mellitus and its complications; Part 1: diagnosis and classification of diabetes mellitus. World Health Organization, Geneva

18. Matthews DR, Hosker JP, Rudenski AS et al (1985) Homeostasis model assessment: insulin resistance and beta-cell function from fasting plasma glucose and insulin concentrations in man. Diabetologia 28:412-419

19. Hotelling H (1931) The generalization of Student's ratio. Ann Math Statist 2:360-378

20. Diabetes Genetics Initiative of Broad Institute of Harvard and MIT, Lund University, and Novartis Institutes of BioMedical Research (2007) Genome-wide association analysis identifies loci for type 2 diabetes and triglyceride levels. Science 316: $1331-1336$ 